\title{
Depuración de procalcitonina en pacientes con respuesta inflamatoria sistémica y su asociación con el desarrollo de delirium
}

\author{
Andrés Santillana Juárez, ${ }^{*}$ María Del Rosario Muñoz Ramírez, ${ }^{*}$ Víctor Manuel Sánchez Nava, ${ }^{*}$ \\ Carlos Eduardo Chávez Pérez, ${ }^{\star}$ Luis Alberto Barrientos Quintanilla, ${ }^{*}$ Pedro Flores Miranda*
}

\section{RESUMEN}

El delirium es una entidad clínica caracterizada por una disfunción aguda en el estado de consciencia con alteraciones de la cognición, atención y curso fluctuante. Se trata de una enfermedad con alta prevalencia en pacientes críticamente enfermos con implicaciones pronósticas. La inflamación sistémica tiene un papel importante en la aparición del delirium y la disfunción cerebra aguda. El objetivo del estudio es buscar una relación entre marcadores clínicos de inflamación, principalmente la depuración de procalcitonina a las 72 horas y su asociación con el desarrollo de delirium en los pacientes de terapia intensiva con síndrome de respuesta inflamatoria sistémica (SIRS). Esto se refleja en los días libres de delirium o coma que el paciente cursa en la unidad de cuidados intensivos (UCI). Se trató de un estudio retrospectivo, en el cual se analizaron 126 pacientes, se observó una mortalidad hospitalaria de $23.01 \%$ y se detectó delirium por CAM-ICU en 69 (55.2\%) de los pacientes. En los pacientes que presentaron delirium se encontraron mayores niveles séricos de procalcitonina al ingreso, a las 24, 48 y 72 horas frente a quienes no lo presentaron. No hubo diferencia entre los niveles de proteína $\mathrm{C}$ reactiva ni diferencias significativas en el porcentaje de depuración de procalcitonina entre los pacientes que presentaron delirium y aquéllos que no. Se detectó un cociente de riesgo de 0.97 (IC $0.96-0.99 ; p=0.0154$ ) por unidad de proteína $C$ reactiva y un cociente de riesgo de 1.17 (IC 1.02-1.35; $p=0.0261$ ) por unidad de aclaramiento de procalcitonina a las 48 horas. Se concluyó que, en pacientes con respuesta inflamatoria sistémica y delirium, algunos marcadores de inflamación están significativamente elevados y representan un factor de riesgo de tener menos días neurológicos normales.

Palabras clave: Delirium, procalcitonina, sepsis, respuesta inflamatoria sistémica, mortalidad, CAM-ICU.

\section{SUMMARY}

Delirium is a clinical entity characterized by an acute disfunction on patients mental state, with altered cognition, inatention, and fluctuating course. Delirium has a high prevalence in critically ill patients, with important prognostic implications. Systemic inflammation plays an important role on the development of delirium and acute cerebral disfunction. The study's objective was to find a correlation between clinical markers of inflammation, specifically procalcitonin clearance, and its association with delirium on intensive care unit patients with Systemic Inflammatory Response Syndrome (SIRS). Studied as a reflection of delirium or coma free days by CAM-ICU that the patient experienced during it's intensive care unit stay. This retrospective study enrolled 126 patients, with a hospital mortality of $23.01 \%$ and a $55.2 \%$ prevalence of delirium as detected by CAM-ICU. Patients with delirium had higher levels of procalcitonin on enrollment as well as at 24, 48 and 72 hours compared to those who did not develop delirium during their ICU stay. There was no difference between $C$ reactive protein or procalcitonin clearance. Higher CRP levels were associated with an decrease in delirium and coma free days (OR 0.97, Cl 0.96-0.99; $p=0.0154$ ), a higher procalcitoin clearance at 48 hours was associated with an increase in delirium and coma free days (OR 1.17, IC 1.02-1.35; $p=0.0261)$. In conclusion, ICU patients with inflammatory response syndrome who develop delirium have a higher risk of developing delirium if inflammatory serum biomarker are elevated, and have a lower chance of having neurologically normal days.

Key words: Delirium, procalcitonin, sepsis, systemic inflammatory response, mortality, CAM-ICU.

\section{RESUMO}

O delirium é uma entidade clínica caracterizada por disfunção aguda no estado de consciência, com alterações na cognição, atenção e curso flutuante. É uma doença com alta prevalência em pacientes em estado crítico com implicações prognósticas. A inflamação sistêmica desempenha um papel importante no aparecimento de delirium e disfunção cerebral aguda. O objetivo deste estudo é buscar uma relação entre os marcadores clínicos de inflamação,

\footnotetext{
* Hospital San José, Tecnológico de Monterrey, Escuela de Medicina y Ciencias de la Salud.
}

Recepción: 01/09/2017. Aceptación: 13/07/2018

Este artículo puede ser consultado en versión completa en http://www.medigraphic.com/medicinacritica principalmente a depuração de procalcitonina às 72 horas, e sua associação com o desenvolvimento de delirium em pacientes em terapia intensiva com síndrome da resposta inflamatória sistêmica (SIRS). Isso é refletido pelos dias sem delirium ou coma que o paciente apresenta na unidade de terapia intensiva. Tratou-se de um estudo retrospectivo em que foram analisados 126 pacientes, encontrando-se uma mortalidade hospitalar de $23.01 \%$ e o delirium detectado pelo CAM-ICU em 69 (55.2\%) dos pacientes. Nos pacientes que apresentaram delirium foram encontrados níveis séricos de procalcitonina mais altos na admissão, às 24, 48 e 72 horas em comparação com aqueles que não a apresentaram. Não houve diferença entre os níveis de proteína C-reativa, nem diferenças significativas na porcentagem de depuração de procalcitonina entre os pacientes que apresentaram delirium e aqueles que não. Foi encontrada uma fator de risco de 0.97 (IC 0.96-0.99, $p=0.0154$ ) por unidade de proteína $C$ reativa e uma fator de risco de 1.17 (IC 1.02-1.35; $p=0.0261$ ) por unidade de depuração da procalcitonina às 48 horas. Concluiu-se que nos pacientes com resposta inflamatória sistêmica e delirium, alguns marcadores de inflamação são significativamente elevados e apresentam um fator de risco para apresentar um número menor de dias neurológicos normais.

Palavras-chave: Delirium, procalcitonina, sepsis, respuesta inflamatória sistêmica, mortalidade, CAM-ICU.

\section{INTRODUCCIÓN}

El delirium es una entidad clínica que se caracteriza por una disfunción aguda en el estado de consciencia por alteraciones de la cognición y la atención con un curso fluctuante. ${ }^{1-3}$ Se trata de una enfermedad con alta prevalencia en pacientes críticamente enfermos, pero con frecuencia pasa desapercibida. ${ }^{4-8}$ El desarrollo de delirium durante la enfermedad crítica tiene implicaciones de pronóstico importantes, pues se asocia a mayor mortalidad y a un deterioro cognitivo en los sobrevivientes. ${ }^{9-12}$ Ésta es la razón por la cual es necesario desarrollar estrategias con el objetivo de identificar de manera oportuna el desarrollo de delirium en la UCI. ${ }^{13}$ Sin embargo, ha sido difícil establecer estrategias para la prevención del delirium y su detección representa un reto en la $\mathrm{UCl}^{2}{ }^{2}$ Una razón por la que el delirium es una enfermedad tan prevalente, representa un reto diagnóstico y un reto mayor de prevención nace del hecho de que se conoce muy poco acerca de su fisiopatología y su relación con la enfermedad crítica. ${ }^{14-18}$ Dentro de la búsqueda de la fisiopatología del delirium, se ha descubierto que existe un enlace entre la inflamación y el desarrollo del delirium, donde niveles elevados de interleucinas proinflamatorias se asocian a mayor desarrollo de delirium en la UCI. ${ }^{19-21}$ La procalcitonina es un marcador de sepsis y tiene un uso bien fundamentado en la práctica clínica actual. Es una herramienta diagnóstica de gran utilidad como guía de la terapia antimicrobiana y marcador pronóstico. ${ }^{22-35}$ Los cambios en los niveles de procalcitonina en el curso de la enfermedad reflejan la respuesta al tratamiento, mientras que el aumento de los niveles séricos de procalcitonina indica un mal 
pronóstico. ${ }^{34-38}$ Existe escasa evidencia de que dicho marcador se encuentre elevado en pacientes con respuesta inflamatoria sistémica que desarrollan delirium, independientemente de la presencia de infección. ${ }^{39,40}$ A pesar de que una procalcitonina elevada al principio de la enfermedad crítica se asocia al desarrollo de delirium, se desconoce si los cambios a través del tiempo también resultarían útiles para identificar a los pacientes con alto riesgo de desarrollar delirium durante su estancia hospitalaria y mejorar la detección temprana del delirium.

\section{MATERIAL Y MÉTODOS}

Se trata de un estudio descriptivo, observacional y retrospectivo, no aleatorizado ni probabilístico. No se realizó ninguna intervención. Se realizó en pacientes con síndrome de respuesta inflamatoria sistémica en la UCI de un hospital privado de la ciudad de Monterrey, N.L. Se trata de una UCI de 14 camas, privada, abierta, con población mixta de pacientes. Se incluyeron pacientes de ambos sexos mayores de 18 años con criterios diagnósticos de síndrome de respuesta inflamatoria sistémica ${ }^{41}$ que ingresaron a la $\mathrm{UCl}$ entre el periodo del 01 de junio de 2014 y el 01 de junio de 2016 y que hubieran cursado más de 72 horas. Se excluyeron pacientes embarazadas o en puerperio inmediato, que hubieran sufrido una patología neurológica en los últimos tres meses, pacientes con diagnóstico probable o confirmado de muerte encefálica, síndrome de postparo cardiorrespiratorio, postoperados de cirugía cardiaca con uso de circulación extracorpórea, SOFA menor de un punto o APACHE II menor de ocho puntos al ingreso y pacientes con un reingreso a UCIA dentro de los últimos tres meses.

Previa aprobación del comité de ética e investigación del hospital, se revisó la base de datos del expediente clínico del hospital en busca de pacientes que cumplieran con los criterios de inclusión ya mencionados y que hubieran ingresado a la UCIA en el periodo especificado. Al ingreso de los pacientes en la UCIA se registran los datos demográficos, tipo de internamiento, así como antecedentes médicos relevantes, deterioro cognitivo preexistente y uso previo de fármacos asociados a delirium. De manera rutinaria, se registran el SOFA y APACHE II al momento del ingreso de todos los pacientes. Los valores de procalcitonina se obtienen al ingreso y a las 24, 48 y 72 horas posteriores. Con base en estos datos, se calcula la depuración de procalcitonina de la siguiente manera:

\section{Procalcitonina inicial-procalcitonina final/ procalcitonina inicial $\times 100^{36,37}$}

También se determinan los niveles de proteína C reactiva dentro de las primeras 24 horas de ingreso.
La medición de procalcitonina y proteína $C$ reactiva se lleva a cabo en el laboratorio del hospital por método de ensayo de electroinmunoluminicencia automatizado. Se valora diariamente el grado de sedación con la escala de RASS y la presencia de delirium o coma detectado por la herramienta CAM-ICU. ${ }^{13}$ Estas variables se miden hasta el egreso de la UCIA o a los 30 días de estancia en dicha unidad. Asimismo, se registra el tipo de sedación, opioides y antipsicóticos, así como su tiempo de uso. Se estudiaron los «días libres de coma o delirium» durante el periodo del estudio, pues de esta manera se refleja el periodo de días en el que el paciente se encontró vivo y libre de disfunción cerebral (delirium o coma); por lo tanto, este parámetro representa los días que el paciente se mantuvo en un estado neurológico «normal» al ser evaluado por el CAM-ICU.42 Del mismo modo, se registran los días de ventilación mecánica, los días de estancia en la UCIA, los días de estancia hospitalaria y la mortalidad.

Las variables de estudio de cada paciente se vaciaron en una base de datos del programa JMP V12.1. De primera intención, se analizó la distribución de las variables utilizando cuentas y porcentajes para la descripción de variables cualitativas, y medias y desviaciones estándar para la descripción de las variables continuas. Para analizar las diferencias entre las variables nominales se utilizó la Prueba de Chi Cuadrada $\left(\chi^{2}\right)$. Para efectuar el análisis entre las diferencias de medias de variables numéricas se empleó la prueba de T para las variables continuas, asumiendo una distribución normal. En caso de que la distribución de las variables fuera asimétrica, se usó la prueba de los rangos con signo de Wilcoxon ( $U$ de Mann-Whitney). Asimismo, se utilizó el modelo de los riesgos proporcionales de Cox para detectar alguna relación entre el riesgo de días libres de delirium/coma, los días de ventilación mecánica, de estancia hospitalaria en la $\mathrm{UCl}$ y las variables independientes. Esta prueba reportó las razones de riesgo con un intervalo de confianza de $95 \%$ para estimar la probabilidad de más o menos días libres de disfunción entre los pacientes en el percentil de $75 \%$ de los niveles de los biomarcadores, comparado con los pacientes en el percentil de $25 \%$ de los niveles de los biomarcadores en la población estudiada. Se analizó si hubo relación lineal o no lineal entre las diferentes variables numéricas y, en su caso, se eliminaron del modelo. Finalmente, se realizó un ajuste de bondad de modelo por regresión logística de las variables continuas en relación con la presencia de delirium y, a partir de esto, se evaluó la habilidad pronóstica de las variables continuas como el área bajo la curva característica operativa del receptor (AUROC). Esta curva ilustra el desempeño de una variable según cambia el umbral de discriminación al representar la razón de verdaderos positivos frente a la razón de falsos positivos (sensibilidad frente a 1-especificidad) de una 
prueba. Para los objetivos de este estudio se consideró una $p \leq 0.05$ como estadísticamente significativa.

\section{RESULTADOS}

Se estudiaron 314 pacientes de ambos sexos mayores de 18 años que ingresaron a la $\mathrm{UCI}$ entre el periodo del 01 de junio de 2014 y el 01 de junio de 2016, con criterios diagnósticos de síndrome de respuesta inflamatoria sistémica y que hayan cursado con una estancia mayor de 72 horas en la UCI. De éstos, se excluyeron del análisis 188 pacientes para un total de 126 pacientes. Las características demográficas de los 126 pacientes analizados se resumen en la Tabla 1. De manera global, se presentó delirium por al menos un día en $55 \%$ de los pacientes, de éstos, $48 \%$ recibieron ventilación mecá-

Tabla 1: Características de la población.

\begin{tabular}{lc}
\hline Variable & $\begin{array}{c}\text { Promedio o cuenta (desviación } \\
\text { estándar o porcentaje) }\end{array}$ \\
\hline Edad & 63.61 años $(18.24)$ \\
Género masculino & $69(55.2)$ \\
Género femenino & $57(45.2)$ \\
Comorbilidades & \\
Ninguna & $18(14.3)$ \\
Hipertensión arterial sistémica & $58(46.0)$ \\
Diabetes mellitus & $52(41.3)$ \\
Insuficiencia renal crónica & $26(20.6)$ \\
Oncológico & $20(15.9)$ \\
Cirrosis hepática & $11(8.7)$ \\
Cardiopatía isquémica & $10(7.9)$ \\
Deterioro cognitivo previo & $24(19)$ \\
Medicamentos asociados a delirium & $38(29.4)$ \\
Tipo de ingreso & \\
Sepsis y choque séptico & $57(45.2)$ \\
Cirugía mayor & $26(20.6)$ \\
Policontindido & $2(1.5)$ \\
Pancreatitis & $3(2.3)$ \\
Quemaduras & $1(0.8)$ \\
Falla respiratoria & $21(0.16)$ \\
Falla cardiaca & $10(7.9)$ \\
Sangrado & $4(3.1)$ \\
Otro & $2(1.5)$ \\
APACHE II & $17.75(7.27)$ \\
SOFA & $7.32(3.43)$ \\
Ventilación mecánica & $61(48.41)$ \\
Delirium & $69(55.2)$ \\
Mortalidad & $29(23.01)$ \\
Días de estancia en UCIA & $8.10(5.56)$ \\
Días de estancia hospitalaria & $17.87(8.58)$ \\
Días de ventilación mecánica & $6.4(5.4)$ \\
Días libres de coma o delirium & $4.57(3.87)$ \\
Tipo de sedación & \\
No & $52(41.3)$ \\
Propofol & $31(24.6)$ \\
Dexmedetomidina & $61(48.4)$ \\
Benzodiacepinas & $24(19.0)$ \\
Días de sedación & $3.47(5.3)$ \\
Uso de opioides & $84(66.6)$ \\
Uso de antipsicóticos & $32(25.39)$ \\
\hline &
\end{tabular}

nica invasiva y la mortalidad hospitalaria fue de $23.01 \%$. El promedio de días de estancia en la UCIA fue de 8.1 días, en los pacientes que recibieron ventilación mecánica el promedio de días de ventilación mecánica fue de 6.4 días y el promedio de estancia hospitalaria fue de 8.6 días. Los niveles promedio de proteína $\mathrm{C}$ reactiva al ingreso fueron de $15.87( \pm 11.16) \mu \mathrm{g} / \mathrm{L}$ y los niveles promedio de procalcitonina al ingreso fue de 26.96 ( \pm 74.69) $\mathrm{ng} / \mathrm{mL}$. Los niveles de procalcitonina disminuyeron en todos los pacientes del momento del ingreso a las 72 horas.

En los pacientes que presentaron delirium se observaron mayores niveles séricos de procalcitonina al ingreso, a las 24,48 y 72 horas frente a quienes no lo presentaron. No hubo una diferencia entre los niveles de proteína $C$ reactiva, ni diferencias significativas en el porcentaje de depuración de procalcitonina entre los pacientes que presentaron delirium y aquéllos que no (Tabla 2 y Figura 1). Sólo los niveles de proteína $C$ reactiva y el aclaramiento de procalcitonina a las 48 horas se asociaron a mayor riesgo de tener menos días libres de delirium o coma por el análisis de riesgos proporcionales de Cox. Se observó un cociente de riesgo de 0.97

Tabla 2: Marcadores de inflamación en pacientes con delirium.

\begin{tabular}{lrcc}
\hline Variable & Sin delirium & Delirium & Valor de $p$ \\
\hline Proteína C reactiva al ingreso $(\mu \mathrm{g} / \mathrm{L})$ & $15.83 \pm 12.87$ & $16.04 \pm 9.67$ & 0.9195 \\
Procalcitonina al ingreso $(\mathrm{ng} / \mathrm{mL})$ & $13.95 \pm 46.90$ & $37.88 \pm 90.58$ & 0.0368 \\
Procalcitonina a las 24 horas $(\mathrm{ng} / \mathrm{mL})$ & $12.28 \pm 44.18$ & $41.26 \pm 93.97$ & 0.0087 \\
Procalcitonina a las 48 horas $(\mathrm{ng} / \mathrm{mL})$ & $12.05 \pm 42.82$ & $31.87 \pm 78.60$ & 0.0092 \\
Procalcitonina a las 72 horas $(\mathrm{ng} / \mathrm{mL})$ & $7.41 \pm 23.00$ & $19.41 \pm 49.76$ & 0.0007 \\
Depuración de procalcitonina & $-20.6 \pm 142.0$ & $-83.2 \pm 248.6$ & 0.0972 \\
a las 24 horas (\%) & $-8.7 \pm 130.8$ & $-53.7 \pm 188.6$ & 0.2090 \\
Depuración de procalcitonina & & & \\
a las 48 horas (\%) & $30.7 \pm 53.2$ & $-55.1 \pm 249.3$ & 0.1799 \\
Depuración de procalcitonina & & & \\
a las 72 horas (\%) & & & \\
\hline
\end{tabular}

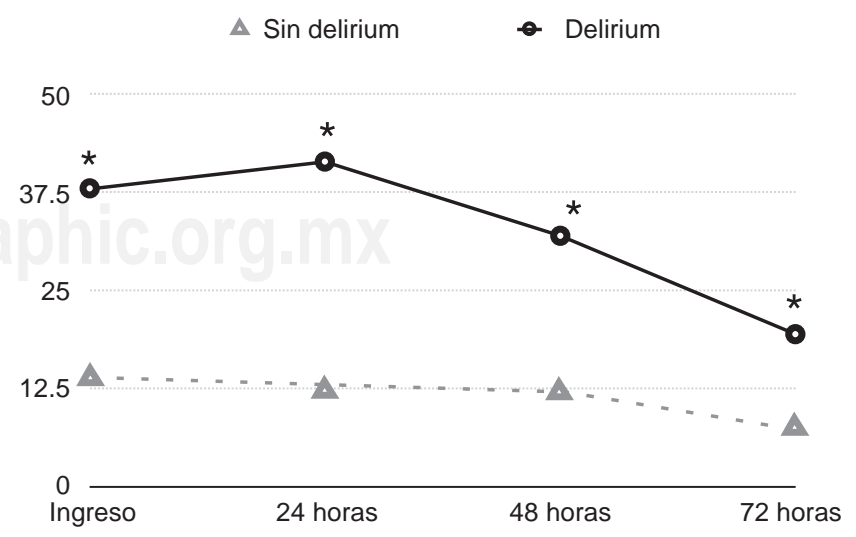

Se grafican las medias de procalcitonina en $\mathrm{ng} / \mathrm{mL}$. ${ }^{*} \mathrm{p}<0.05$ pacientes que presentaron delirium frente a quienes no lo presentaron.

Figura 1: Niveles de procalcitonina a través del tiempo en delirium. 
(IC 0.96-0.99; $p=0.0154$ ) por unidad de proteína $C$ reactiva y un cociente de riesgo de 1.17 (IC 1.02-1.35; p $=0.0261$ ) por unidad de aclaramiento de procalcitonina a las 48 horas. Esto significa que cada aumento de unidad con respecto a la unidad previa (por ejemplo de $0 \mu \mathrm{g} / \mathrm{L}$ a $1 \mu \mathrm{g} / \mathrm{L}$ ) en los niveles séricos de proteína $C$ reactiva confiere menos posibilidades de tener días libres de delirium o coma. En el caso del aclaramiento de procalcitonina a las 48 horas, un aumento de la unidad con respecto a la unidad previa (por ejemplo de $0 \%$ al $100 \%$ ) confiere más posibilidades de tener días libres de delirium o coma, recordando que los días libres de delirium o coma representan días «normales» a nivel neurológico. Finalmente, por un modelo de regresión logística, las variables con mejor discriminación para predecir la presencia de delirium fueron la depuración de procalcitonina a 72 horas y el puntaje APACHE II. La depuración de procalcitonina a 72 horas presentó un área bajo la curva ROC de 0.56988 con una $p=0.0177$, demostrando un punto de corte de $-91.36 \%$ de depuración. El puntaje APACHE II reveló un área bajo la curva ROC de 0.65463 con una $p=0.0065$, demostrando un punto de corte de 18 puntos (Figura 2).

\section{ANÁLISIS Y CONCLUSIONES}

Analizando específicamente la asociación entre la proteína $C$ reactiva y la presencia de delirium, este estudio difiere en algunos puntos con el resto de la literatura que aborda el tema. Como tal, en la población estudiada no se demostraron niveles séricos de procalcitonina más altos al ingreso en los pacientes que presentaron delirium, frente a quienes no lo presentaron. ${ }^{43,44}$ Este hallazgo contrasta con estudios previos que sí han demostrado una diferencia en los niveles séricos de proteína $C$ reactiva al ingreso, por ejemplo, Zhang y cols. efectivamente detectaron una diferencia en los niveles de proteína $C$ reactiva de ingreso en los pacientes que desarrollaron delirium (120.5 versus $57.5 \mathrm{mg} / \mathrm{L} ; p$ $=0.0001$ ), independiente de otras variables de severidad. ${ }^{43}$ Esto puede deberse a la naturaleza del estudio, pues fue un análisis prospectivo, mientras que el presente análisis fue retrospectivo $\mathrm{y}$, como tal, no hubo un protocolo para la recolección de las variables. Por su parte, Macdonald y cols. no observaron una diferencia en niveles al ingreso de proteína $C$ reactiva en los niveles en pacientes que ya presentaban delirium al ingreso frente a quienes no lo presentaban, aunque los niveles de proteína $C$ reactiva que se encontraban elevados se asociaron a la recuperación de un episodio de delirium previo o a la incidencia de delirium. ${ }^{45}$ Sin embargo, este estudio se enfocó más en los niveles de proteína $\mathrm{C}$ reactiva y su asociación de riesgo de tener días libres de coma o delirium; en este aspecto, el análisis fue similar al estudio de McGrane y cols, ${ }^{42}$ en el que evaluaron la
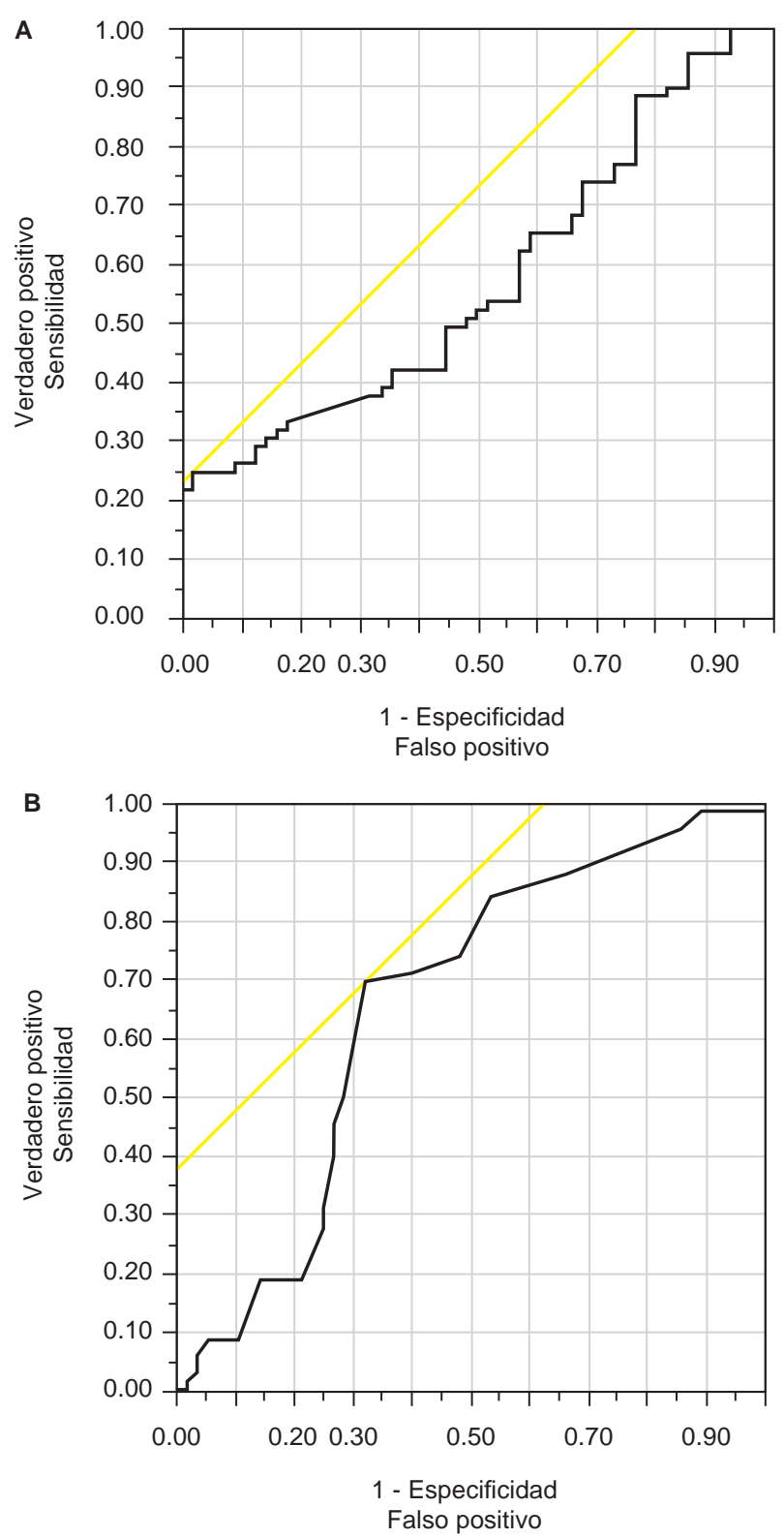

Se grafica el área bajo la curva característica operativa del receptor. A: Depuración de procalcitonina a las 72 horas. B: APACHE II.

Figura 2: Curvas ROC para predicción de delirium.

probabilidad de tener un día libre de delírum o coma con base en los niveles al ingreso de proteína $C$ reactiva; esto es, la probabilidad de tener días neurológicos «normales», encontrando que niveles más altos de proteína $C$ reactiva reducían significativamente la probabilidad de tener días libres de delirium o coma (OR de 0.6 ; IC 95\%, 0.3 a 1.1; P = 0.08). De esta manera, los hallazgos son similares a los del presente estudio, pues se demostró un cociente de riesgo (OR) de 0.97 (IC 0.96-0.99; $p=0.0154$ ) por unidad de proteína $C$ reactiva. Esto significa que cada aumento de unidad con respecto a la unidad previa (por ejemplo de $0 \mu \mathrm{g} / \mathrm{L}$ fren- 
te a $1 \mu \mathrm{g} / \mathrm{L}$ o de $1 \mu \mathrm{g} / \mathrm{L}$ frente a $2 \mu \mathrm{g} / \mathrm{L}$ ) en los niveles séricos de proteína $\mathrm{C}$ reactiva confiere menos posibilidades de tener días libres de delirium o coma. Dicho de una manera más simple, un mayor nivel de proteína $C$ reactiva al ingreso disminuye las posibilidades de que los pacientes tengan días neurológicamente normales. Es probable que la diferencia en el cociente de riesgo entre este estudio y el trabajo de McGrane se deba a que dicho trabajo evaluó los cambios por cambio en cuantiles de los niveles de proteína $C$ reactiva y no los cambios en las unidades absolutas, como este estudio.

En relación con las determinaciones individuales de procalcitonina, se observó una diferencia significativa en los niveles séricos entre cada una de ellas en los pacientes que desarrollaron delirium frente a quienes no lo desarrollaron. Fue similar a la investigación de van den Boogard y cols., en la que se detectaron diferencias entre los niveles séricos de procalcitonina al ingreso en pacientes que desarrollaron delirium y quienes no lo desarrollaron $(0.35 \mathrm{ng} / \mathrm{mL}$ versus $0.14 \mathrm{ng} / \mathrm{mL} ; \mathrm{p}<0.001)$, aunque en una proporción mayor en el presente estudio. ${ }^{40}$ Probablemente, esto se deba a una proporción más alta de pacientes sépticos y a mayor gravedad de la enfermedad aguda. Cabe mencionar que el estudio que nos ocupa ha evaluado los niveles de procalcitonina a través del tiempo y no se observaron diferencias importantes en la tasa de depuración de procalcitonina entre los pacientes que desarrollaron delirium y aquéllos que no. Los niveles individuales de procalcitonina o los cálculos de depuración de procalcitonina tampoco resultaron útiles para la predicción de desarrollo del delirium; sólo la depuración de procalcitonina a 72 horas presentó una área bajo la curva ROC de 0.56988 con una $p=0.0177$, demostrando un punto de corte de $-91.36 \%$ de depuración, lo cual fue significativamente menor que el puntaje APACHE II. Pese a ello, en el análisis de riesgos proporcionales, la depuración de procalcitonina a las 48 horas demostró un cociente de riesgo (OR) de 1.17 (IC 1.02 - 1.35; $p=0.0261$ ) por cada $100 \%$ de aclaramiento de procalcitonina. Esto significa que un aumento de aclaramiento de procalcitonina de 0 a $100 \%$ confiere un $17 \%$ más posibilidades de presentar días libres de delirium o coma. En otras palabras, un mayor porcentaje de aclaramiento de procalcitonina a las 48 horas aumenta las posibilidades de que los pacientes tengan días neurológicamente normales. Contrario al estudio de McGrane y cols., previamente mencionado, en los niveles de procalcitonina al ingreso no se observó un cociente menor de riesgo de tener días libres de coma o delirium. La razón de dichos hallazgos podría tener varias explicaciones, es posible que el aclaramiento de procalcitonina tenga mejor relación con los días libres de delirium o coma y, por lo tanto, los niveles individuales de procalcitonina no hayan resultado significativos. Como se señaló anteriormente, los niveles de procalcitonina en este estudio fueron significativamente mayores que en otras investigaciones similares y es probable que se deba a la naturaleza retrospectiva del estudio, comparado con el trabajo de McGrane, que recolectó sus datos de manera prospectiva dentro de un ensayo clínico controlado. ${ }^{46}$

Finalmente, cabe mencionar los hallazgos respecto a la mortalidad del estudio, donde sólo se observó una diferencia entre los porcentajes de depuración de procalcitonina a las 72 horas entre los pacientes que sobrevivieron y los que fallecieron dentro del hospital, similar a lo demostrado en investigaciones previas, aunque por análisis de regresión logística todas las variables asociadas a la procalcitonina no demostraron discriminación en la predicción de mortalidad en la población estudiada de pacientes críticamente enfermos. ${ }^{35-37}$ Estas diferencias se atribuyen nuevamente a la naturaleza retrospectiva del estudio y es probable que el médico tratante no haya tomado determinaciones de procalcitonina en pacientes con menor gravedad de la enfermedad o cuando no haya existido una incertidumbre diagnóstica y, por lo tanto, esto haya sesgado de alguna manera las mediciones del biomarcador en el presente estudio. También se considera que la posibilidad de dichos hallazgos se deba a que estos resultados han sido derivados de pacientes exclusivamente con choque séptico y no de poblaciones de pacientes que no han sido sometidos a una selección más rigurosa, como en el estudio que nos ocupa, cuyos objetivos se enfocaron en pacientes con estados proinflamatorios más variados y en excluir los factores confusos en la fisiopatología y en la detección de delirium.

Como se ha mencionado, este estudio tiene varías limitaciones debido al diseño descriptivo y recolección retrospectiva de los datos. La recolección de los datos está a expensas de lo que se ha registrado previamente en el expediente clínico y es posible que los datos relevantes para el estudio no hayan sido registrados. Desafortunadamente, tampoco es posible obtener o corroborar los datos faltantes. También existe la posibilidad de que los datos registrados no sean los correctos o no correspondan al paciente. Además de los problemas inherentes del registro adecuado de datos, las decisiones clínicas no seguían un protocolo específico predeterminado, estaban sujetas al escenario clínico del momento en el que se presentaron, a la interpretación clínica de los datos por los médicos, así como a las preferencias de los tratantes. Por lo tanto, el estudio sólo se limita a describir las asociaciones y no puede evaluar de manera adecuada las intervenciones realizadas ni su efecto en los niveles de procalcitonina. Hubiera sido deseable, también, dar seguimiento a los pacientes por más tiempo, como ha sido en estudios previos, hasta el año del egreso hospitalario, para evaluar el grado de discapacidad neurológica o incluso mortalidad a largo plazo. 
Por el momento, con base en la evidencia científica actual y los resultados del presente trabajo, puede concluirse que la inflamación sistémica representa un factor de riesgo de padecer delirium en la UCI. No es posible definir si existe una relación causa-efecto entre los niveles elevados de biomarcadores y el desarrollo del delirium o si sólo representan un marcador de severidad. Aún faltan estudios que aclaren si esta asociación tendrá alguna utilidad clínica en el desarrollo de estrategias para la prevención, detección o tratamiento del delirium.

\section{BIBLIOGRAFÍA}

1. Girard TD, Pandharipande PP, Ely EW. Delirium in the intensive care unit. Crit Care. 2008;12(Suppl 3):S3.

2. Jones SF, Pisani MA. ICU delirium: an update. Curr Opin Crit Care. 2012;18(2):146-151.

3. American Psychiatric Association. Diagnostic and statistical manual of mental disorders: text revision. 5th ed. American Psychiatric Association, Washington, D.C. 2013.

4. Morandi A, Pandharipande P, Trabucchi M, Rozzini R, Mistraletti G, Trompeo AC, et al. Understanding international differences in terminology for delirium and other types of acute brain dysfunction in critically ill patients. Intensive Care Med. 2008;34(10):1907-1915.

5. Ely EW, Shintani A, Truman B, Speroff T, Gordon SM, Harrell FE Jr., et al. The impact of delirium in the intensive care unit on hospital length of stay. Intensive Care Med. 2001;27(12):1892-1900.

6. Ely EW, Shintani A, Truman B, Speroff T, Gordon SM, Harrell FE Jr., et al. Delirium as a predictor of mortality in mechanically ventilated patients in the intensive care unit. JAMA. 2004;291(14):1753-1762.

7. Dubois MJ, Bergeron N, Dumont M, Dial S, Skrobik Y. Delirium in an intensive care unit: a study of risk factors. Intensive Care Med. 2001;27(8):1297-1304.

8. McNicoll L, Pisani MA, Zhang Y, Ely EW, Siegel MD, Inouye SK. Delirium in the intensive care unit: occurrence and clinical course in older patients. J Am Geriatr Soc. 2003;51(5):591-598.

9. Girard TD, Jackson JC, Pandharipande PP, Pun BT, Thompson $\mathrm{JL}$, Shintani AK, et al. Delirium as a predictor of long-term cognitive impairment in survivors of critical illness. Crit Care Med. 2010;38(7):1513-1520.

10. Pandharipande PP, Girard TD, Jackson JC, Morandi A, Thompson JL, Pun BT, et al. Long-term cognitive impairment after critical illness. N Engl J Med. 2013;369(14):1306-1316.

11. Salam A, Tilluckdharry L, Amoateng-Adjepong Y, Manthous CA. Neurologic status, cough, secretions and extubation outcomes. Intensive Care Med. 2004;30(7):1334-1339.

12. Milbrandt EB, Deppen S, Harrison PL, Shintani AK, Speroff T, Stiles RA, et al. Costs associated with delirium in mechanically ventilated patients. Crit Care Med. 2004;32(4):955-962.

13. Barr J, Fraser GL, Puntillo K, Ely EW, Gélinas C, Dasta JF, et al. Clinical practice guidelines for the management of pain, agitation, and delirium in adult patients in the intensive care unit. Crit Care Med. 2013;41(1):263-306.

14. Hughes CG, Patel MB, Pandharipande PP. Pathophysiology of acute brain dysfunction: what's the cause of all this confusion? Curr Opin Crit Care. 2012;18(5):518-526.

15. Jackson $P$, Khan A. Delirium in critically ill patients. Crit Care Clin. 2015;31(3):589-603.

16. Hughes CG, Morandi A, Girard TD, Riedel B, Thompson JL, Shintani AK, et al. Association between endothelial dysfunction and acute brain dysfunction during critical illness. Anesthesiology. 2013;118(3):631-639.

17. Ebersoldt M, Sharshar T, Annane D. Sepsis-associated delirium. Intensive Care Med. 2007;33(6):941-950.

18. Grandi C, Tomasi CD, Fernandes K, Stertz L, Kapczinski F, Quevedo J, Dal-Pizzol F, et al. Brain-derived neurotrophic factor and neuron-specific enolase, but not S100ß, levels are associated to the occurrence of delirium in intensive care unit patients. J Crit Care. 2011;26(2):133-137.

19. de Rooij SE, van Munster BC, Korevaar JC, Levi M. Cytokines and acute phase response in delirium. J Psychosom Res. 2007;62(5):521-525.

20. van Munster BC, Bisschop PH, Zwinderman AH, Korevaar JC, Endert E, Wiersinga WJ, et al. Cortisol, interleukins and S100B in delirium in the elderly. Brain Cogn. 2010;74(1):18-23.

21. Adamis D, Lunn M, Martin FC, Treloar A, Gregson N, Hamilton $\mathrm{G}$, et al. Cytokines and IGF-I in delirious and non-delirious acutely ill older medical inpatients. Age Ageing. 2009;38(3):326332.

22. Uzzan B, Cohen R, Nicolas P, Cucherat M, Perret GY. Procalcitonin as a diagnostic test for sepsis in critically ill adults and after surgery or trauma: a systematic review and metaanalysis. Crit Care Med. 2006;34(7):1996-2003.

23. Tang BM, Eslick GD, Craig JC, McLean AS. Accuracy of procalcitonin for sepsis diagnosis in critically ill patients: systematic review and meta-analysis. Lancet Infect Dis. 2007;7(3):210-217.

24. Wacker C, Prkno A, Brunkhorst FM, Schlattmann P. Procalcitonin as a diagnostic marker for sepsis: a systematic review and metaanalysis. Lancet Infect Dis. 2013;13(5):426-435.

25. Schuetz $P$, Müeller B. Procalcitonin in critically ill patients: time to change guidelines and antibiotic use in practice. Lancet Infectious Diseases. 2016;16(7):758-760.

26. Schuetz P, Chiappa V, Briel M, Greenwald JL. Procalcitonin algorithms for antibiotic therapy decisions: a systematic review of randomized controlled trials and recommendations for clinical algorithms. Arch Intern Med. 2011;171(15):1322-1331.

27. Bouadma L, Luyt CE, Tubach F, Cracco C, Alvarez A, Schwebel $C$, et al. Use of procalcitonin to reduce patients' exposure to antibiotics in intensive care units (PRORATA trial): a multicentre randomised controlled trial. Lancet. 2010;375(9713):463-474.

28. de Jong E, van Oers JA, Beishuizen A, Vos P, Vermeijden WJ, Haas LE, et al. Efficacy and safety of procalcitonin guidance in reducing the duration of antibiotic treatment in critically ill patients: a randomised, controlled, open-label trial. Lancet Infect Dis. 2016;16(7):819-827.

29. Prkno A, Wacker C, Brunkhorst FM, Schlattmann P. Procalcitonin-guided therapy in intensive care unit patients with severe sepsis and septic shock--a systematic review and metaanalysis. Crit Care. 2013;17(6):R291.

30. Soni NJ, Samson DJ, Galaydick JL, Vats V, Huang ES, Aronson $\mathrm{N}$, et al. Procalcitonin- guided antibiotic therapy: a systematic review and meta-analysis. J Hosp Med. 2013;8(9):530-540.

31. Afsar I, Sener AG. Is procalcitonin a diagnostic and/or prognostic marker in sepsis? Infectious Diseases in Clinical Practice. 2015;23(1):3-6.

32. Kibe S, Adams K, Barlow G. Diagnostic and prognostic biomarkers of sepsis in critical care. J Antimicrob Chemother. 2011;66(Suppl 2):ii33-ii40.

33. Jensen JU, Heslet L, Jensen TH, Espersen K, Steffensen $\mathrm{P}$, Tvede M. Procalcitonin increase in early identification of critically ill patients at high risk of mortality. Crit Care Med. 2006;34(10):2596-2602.

34. Jain S, Sinha S, Sharma SK, Samantaray JC, Aggrawal P, Vikram NK, et al. Procalcitonin as a prognostic marker for sepsis: a prospective observational study. BMC Res Notes. 2014;7:458.

35. Ruiz-Rodriguez JC, Caballero J, Ruiz-Sanmartin A, Ribas VJ, Pérez M, Bóveda JL, et al. Usefulness of procalcitonin clearance as a prognostic biomarker in septic shock. A prospective pilot study. Med Intensiva. 2012;36(7):475-480.

36. Suberviola B, Castellanos-Ortega A, González-Castro A, García-Astudillo LA, Fernández-Miret B. Valor pronóstico del aclaramiento de procalcitonina, PCR y leucocitos en el shock séptico. Med Intensiva. 2012;36(3):177-184.

37. de Azevedo JR, Torres OJ, Beraldi RA, Ribas CA, Malafaia O. Prognostic evaluation of severe sepsis and septic shock: procalcitonin clearance vs $\Delta$ Sequential Organ Failure Assessment. J Crit Care. 2015;30(1):219.e9-12. 
38. Hatzistilianou M, Rekleity A, Athanassiadou F, DeLutiis MA, Conti P, Catriu D. Serial procalcitonin responses in infection of children with secondary immunodeficiency. Clin Invest Med. 2007;30(2):E75-85.

39. van den Boogaard M, Kox M, Quinn KL, van Achterberg T, van der Hoeven JG, Schoonhoven L, et al. Biomarkers associated with delirium in critically ill patients and their relation with longterm subjective cognitive dysfunction; indications for different pathways governing delirium in inflamed and noninflamed patients. Crit Care. 2011;15(6):R297.

40. Kazmierski J, Banys A, Latek J, Bourke J, Jaszewski R. Cortisol levels and neuropsychiatric diagnosis as markers of postoperative delirium: a prospective cohort study. Crit Care. 2013;17(2):R38.

41. Levy MM, Fink MP, Marshall JC, Abraham E, Angus D, Cook D, et al. 2001 SCCM/ESICM/ACCP/ATS/SIS International sepsis definitions conference. Crit Care Med. 2003;31(4):1250-1256.

42. McGrane S, Girard TD, Thompson JL, Shintani AK, Woodworth A, Ely EW, et al. Procalcitonin and C-reactive protein levels at admission as predictors of duration of acute brain dysfunction in critically ill patients. Crit Care. 2011;15(2):R78.

43. Macdonald A, Adamis D, Treloar A, Martin F. C-reactive protein levels predict the incidence of delirium and recovery from it. Age Ageing. 2007;36(2):222-225.
44. Zhang Z, Pan L, Deng $\mathrm{H}, \mathrm{Ni} \mathrm{H}, \mathrm{Xu} X$. Prediction of delirium in critically ill patients with elevated C-reactive protein. J Crit Care. 2014;29(1):88-92.

45. Ritchie CW, Newman TH, Leurent B, Sampson EL. The association between $\mathrm{C}$-reactive protein and delirium in 710 acute elderly hospital admissions. Int Psychogeriatr. 2014;26(5):717724.

46. Pandharipande PP, Pun BT, Herr DL, Maze M, Girard TD, Miller $\mathrm{RR}$, et al. Effect of sedation with dexmedetomidine vs lorazepam on acute brain dysfunction in mechanically ventilated patients: the MENDS randomized controlled trial. JAMA. 2007;298(22):26442653.

Correspondencia:

Andrés Ulises Santillana Juárez Morones Prieto Núm. 3000 pte,

Col. Los Doctores, 64710,

Monterrey, Nuevo León.

Tel: (81) 83475828

E-mail: santillana.e@gmail.com 